\title{
Stellar dynamics and tidal disruption events in galactic nuclei
}

\author{
T. Alexander ${ }^{\mathrm{a}}$ \\ Weizmann Institute of Science, Faculty of Physics, Department of Particle Physics and Astrophysics
}

\begin{abstract}
The disruption of a star by the tidal field of a massive black hole is the final outcome of a chain of complex dynamical processes in the host galaxy. I introduce the "loss cone problem", and describe the many theoretical and numerical challenges on the path of solving it. I review various dynamical channels by which stars can be supplied to a massive black hole, and the relevant dynamical relaxation/randomization mechanisms. I briefly mention some "exotic" tidal disruption scenarios, and conclude by discussing new dynamical results that are changing our understanding of dynamics near a massive black hole, and may well be relevant for tidal disruption dynamics.
\end{abstract}

\section{OUTLINE}

This is a brief, informal review of the stellar dynamical mechanisms that play a role in the tidal destruction (TD) of stars by massive black holes (MBHs) in galactic nuclei. The key issue is to determine how, and at what rate, are stars deflected to the extremely eccentric orbits that bring them sufficiently close to the MBH for TD. This is the so called "loss-cone (LC) replenishment problem", which is introduced in its most basic form (single stars around a single $\mathrm{MBH}$, scattered singly into the LC) in Sec. 2, together with a discussion of the more general framework of infall/inspiral processes, and other channels of LC replenishment. Orbital evolution (denoted here generally by the term "relaxation"), whether stochastic or coherent, can take various forms and proceed at a wide range of rates on different spatial scales. Some lesser known forms of relaxation are presented in Sec. 3, and their impact on TD is discussed. TD rate estimates, and the rates of associated "exotic" processes, are reviewed in Sec. 4. Sec. 5 explores some interesting new dynamical processes that may be relevant for stars that are formed or captured very close to the $\mathrm{MBH}$, and deflected to the LC from tight orbits. Sec. 6 briefly summarizes the main conclusions and offers a few final comments.

\section{GETTING STARS TO THE MBH: THE LOSS CONE}

\subsection{Basic results}

TD occurs when a star approaches an $\mathrm{MBH}$ of mass $M_{\bullet}$ closer than the TD radius $r_{t} \simeq R_{\star}\left(M_{\bullet} / M_{\star}\right)^{1 / 3}$, where $R_{\star}$ and $M_{\star}$ are the star's radius and mass. Alternative physical formulations of the TD criterion are that the star is destroyed when its typical density, $\rho_{\star} \sim M_{\star} / R_{\star}^{3}$, falls below the density the MBH would have had if its mass were spread over the volume $r_{t}^{3}$, or that the star is destroyed when the crossing time through the disruption zone $\left(r_{t}^{3} / G M_{\bullet}\right)^{1 / 2}$ falls below the star's free-fall time $\left(R_{\star}^{3} / G M_{\star}\right)^{1 / 2}$. TD by a MBH can occur only when $r_{t}$ lies

\footnotetext{
a e-mail: tal.alexander@weizmann.ac.il
}

outside the event horizon at $r_{e}=x G M_{\bullet} / c^{2}$, where $x=2$ for a non-spinning $\mathrm{MBH}$, and can be as small as $x=1$ for a maximally spinning $\mathrm{MBH}$ and a co-orbiting star. Since $r_{t} / r_{e} \propto \rho_{\star}^{-1 / 3} x^{-1} M_{\bullet}^{-2 / 3}$, it follows that the less massive the $\mathrm{MBH}$, the more compact the stars that undergo TD can be; for a star with given $R_{\star}, M_{\star}$, there exists a maximal $\mathrm{MBH}$ mass, $\max M_{\bullet}$, where TD is still possible; and the higher the $\mathrm{MBH}$ spin, the larger is $\max M_{\text {. }}$.

A star on an orbit with specific energy $E=G M_{\bullet} / 2 a$ and specific angular momentum $J=J_{c} \sqrt{1-e^{2}}$ (The Keplerian limit is assumed, with the convention that $E=$ $-E_{\text {true }}>0$ for a bound orbit. $a$ is the semi-major axis (sma), $e$ the eccentricity, and $J_{c}=\sqrt{G M_{\bullet} a}$ is the specific circular angular momentum) will reach periapse inside the $\mathrm{TD}$ radius, $r_{p}=a(1-e) \leq r_{t}$ if its angular momentum is less than the LC angular momentum $J_{l c}=$ $\sqrt{G M_{\bullet} r_{t}(1+e)}$ (Fig. 1L). All stars that are initially on LC orbits will be destroyed in less than an orbital period $P$. From that time on, the rate of TD events will depend on the rate at which dynamical mechanisms repopulate these orbits.

The discreteness of a stellar system guarantees a minimal orbital randomization rate by incoherent 2-body relaxation, on the timescale $t_{E} \sim Q^{2} P / N_{\star} \log Q$, where $Q=M_{\bullet} / M_{\star}$ and $N_{\star}(a)$ is the number of stars with sma $\leq a$ (here and below I informally interchange $r \leftrightarrow a$ ). This is the time it takes the orbital energy to change by order unity. Because the 2-body scattering is local and isotropic (i.e. it does not "care" where the MBH is), the time for an order unity change in angular momentum can be much shorter than $t_{E}$ if the orbit already has low angular momentum, $t_{J} \sim\left[J / J_{c}(E)\right]^{2} t_{E}$. It then follows that stars get deflected to LC orbits primarily by relaxation of angular momentum, and not energy (Fig. 1C).

More detailed calculations [e.g. [1]] indicate that the total TD rate is $\Gamma_{\mathrm{TD}} \sim N_{\star}\left(<r_{h}\right) /\left\langle\log \left(J_{c}(a) / J_{l c}\right) t_{E}(a)\right\rangle$, where $r_{h}$ is the MBH's radius of influence, $r_{h} \sim G M_{\bullet} / \sigma^{2}$, where $\sigma$ is the velocity dispersion far from the $\mathrm{MBH}$, and where the average $\langle\cdots\rangle$ is over the volume within $r_{h}$. Note that the dependence of the rate on the relative size of the LC is only logarithmic, so that the TD rate scales approximately as the number of stars in the relevant reservoir, over the relaxation time on that scale. 

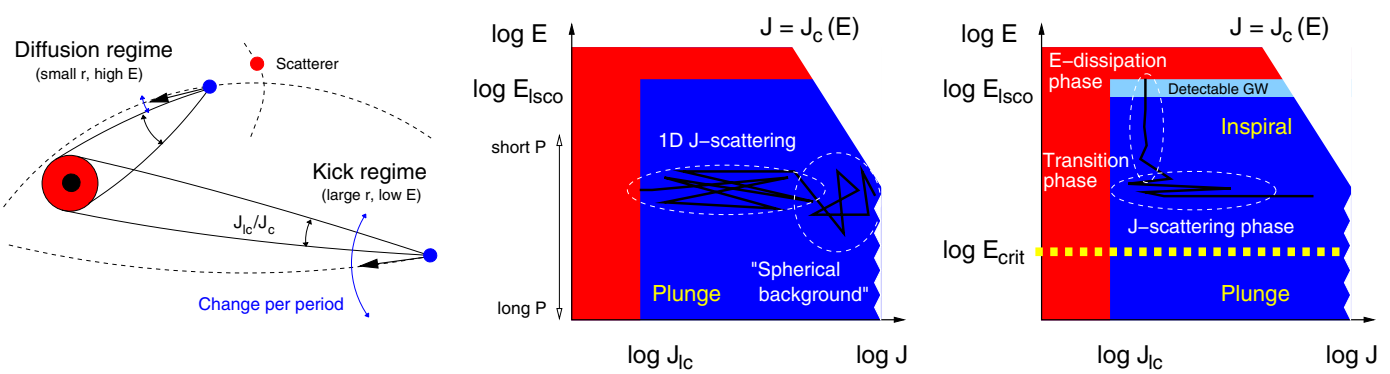

Figure 1. Schematic depiction of LC dynamics in real space and in phase space, and of the difference between infall and inspiral. Left: a test star's velocity vector is deflected into the LC by an interaction with a field star (scatterer). When the test star is relatively close to the $\mathrm{MBH}$, the angular opening of the LC is large, while the magnitude of a typical accumulated deflection per orbit is small; stars diffuse into the $\mathrm{LC}$ at a rate much slower than the time it takes a star to reach the $\mathrm{MBH}$, and the $\mathrm{LC}$ is almost empty of stars. Conversely, for stars far from the MBH, the angular size is smaller than the typical accumulated deflection per orbit, stars are rapidly "kicked" into the middle of the LC, which remains almost full. In typical galactic nuclei, most tidally disrupted stars originate from the transition range between the empty and full LC regimes, at $r \sim r_{h}$, where $N_{\star} M_{\star} \sim M_{\bullet}$. Center: the phase space $(\log J, \log E$ ) of infall (plunge) events, such as tidal disruption, which happen promptly once the star gets close enough to the MBH. Since $t_{J} \sim\left(J / J_{c}\right)^{2} t_{E}$, once a star reaches a somewhat eccentric orbit, there is a substantial probability that it will be scattered into the LC and promptly destroyed. Right: the behavior in the presence of a dissipative process, for example the emission of gravitational waves $(\mathrm{GW})$, whose luminosity sharply increases with proximity to the $\mathrm{MBH}$. Close enough to the $\mathrm{MBH}$, above some critical energy $E_{\text {crit }}$, (equivalently, inside some critical radius $r_{\text {crit }}$ ), where the dissipation per period is strong, orbital decay is faster that the 2-body relaxation, and almost all stars eventually inspiral gradually into the MBH. Conversely, outside that region, almost all stars ultimately plunge into the MBH.

\subsection{Plunge vs. inspiral}

TD is one example of an infall process, where the event of interest occurs promptly once the star reaches close enough to the $\mathrm{MBH}$. It is instructive to consider also the related class of inspiral processes, where energy is dissipated gradually on successive peripassages, and the orbit decays until the star is destroyed. The dissipation can be, for example, by tidal heating (Sec. 4.3), or by the emission of gravitational waves $(\mathrm{GW})$. In both cases the dissipated power is a strong function of the distance from the $\mathrm{MBH}$, and therefore almost all of it occurs near periapse. Since inspiral is a race between deterministic dissipation and stochastic scattering, there exists a boundary, which is approximately constant in energy (equivalently, a typical radius from the $\mathrm{MBH}$ ), that separates infall from inspiral (Fig.1R). Far from the $\mathrm{MBH}$, where the orbital time is long, there is enough time between peripassages to be scattered off the nearly radial orbit required for a close encounter with the $\mathrm{MBH}$. Therefore stars beyond some critical radius $r_{\text {crit }}$ ultimately fall directly (plunge) into the $\mathrm{MBH}$. Conversely, stars inside $r_{\text {crit }}$ inspiral gradually into the $\mathrm{MBH}$, at a rate $\Gamma_{\text {inspiral }} \sim N_{\star}\left[<r_{\text {crit }}\left(t_{E}\right)\right] /\left\langle\log \left(J_{c} / J_{l c}\right) t_{E}\right\rangle$. This expression, whose interpretation is similar to that of the infall rate, is deceptively simple, since it is non-linear because $r_{\text {crit }}$ depends itself on $t_{E}$. For GW inspiral in the GC, $r_{\text {crit }} \sim$ $O(0.01 \mathrm{pc})$ (Fig. 2R). Because typically $r_{\text {crit }} \ll r_{h}$, and the number of stars enclosed within $r_{\text {crit }}$ is much smaller than in $r_{h}$, it is generally the case that $\Gamma_{\text {inspiral }} \sim O(0.01) \Gamma_{\text {plunge }}$.

\subsection{The challenges}

Many conceptual, technical, and astrophysical challenges stand in the way of reliable theoretical predictions of TD rates and their parameter dependences. These issues and open questions broadly fall in three categories:
Analytic representation. How to achieve a tractable self-consistent description of the stellar system? How to set boundary conditions correctly? How to describe the diffusion of orbital elements in phase space?

Numeric simulation. How to simulate the $N_{\star} \rightarrow \infty$ limit? How to deal with the $r \rightarrow 0$ limit (very close encounters; General Relativistic (GR) effects)?

Galactic models. Which are the dominant components (e.g. one or two MBHs; gas; disks)? What stellar population to assume (mass spectrum)? What stellar density to assume (distribution function)? What symmetries to assume (e.g. spherical, axial)?

While dealing with questions of analytic representation and numeric simulation may require sophisticated analysis and state of the art computational techniques, these issues are in principle solvable, even if only by brute force computation. In contrast, the situation with the astrophysical uncertainties of galactic modeling presents an irreducible problem, since it is unlikely that these could be determined with any degree of certainty in the foreseeable future. At this time there is no recourse but to explore a large parameter space of plausible scenarios, and map out the range of possible outcomes.

\subsection{Supply channels}

Although much of the theoretical effort of modeling TD events focused on the simplest configuration: stars that are singly deflected toward a single $\mathrm{MBH}^{1}$ (Sec. 4.1), there are quite a few additional possible channels leading to TD events. These include binary stars separated by the tidal

\footnotetext{
${ }^{1}$ This dynamical configuration also applies to stars and white dwarfs disrupted by intermediate mass black holes (IMBHs), or comets disrupted by a central object.
} 

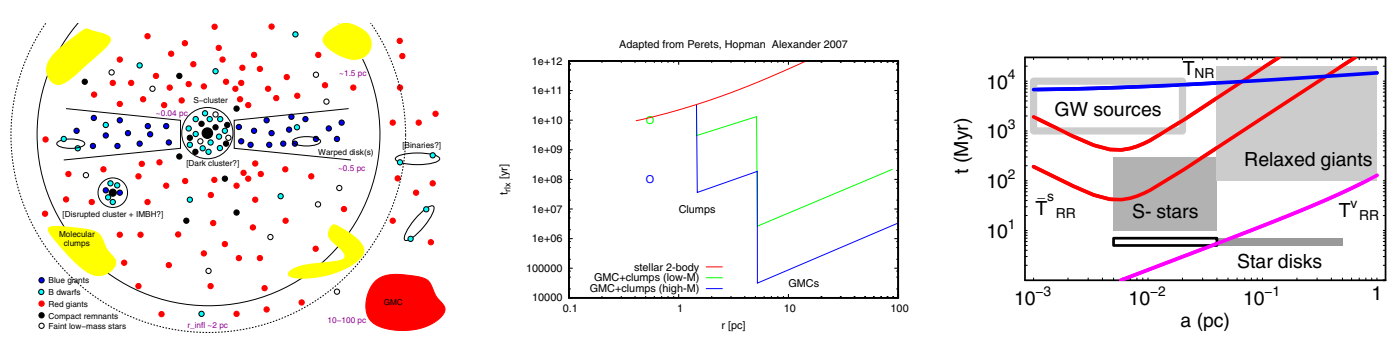

Figure 2. Lessons from the GC. Left: a not-to-scale schematic of the various dynamical components that are observed in the GC around $\mathrm{SgrA}^{\star}$, or are hypothesized to exist there (marked by [...]) [8]. Note in particular the massive GMCs and the circum-nuclear molecular disk at or outside the MBH's radius of influence; the mixed old and young stellar population inside $r_{h}$ (only the giants and B-dwarfs are above the present-day detection threshold); the $O(100)$ blue giants that are concentrated in one (or perhaps two) coherently-rotating warped disks, which extend inward to a sharp inner cut-off at $\sim 0.04 \mathrm{pc}(\sim 1$ "); and the spectroscopically and dynamically distinct population of $\sim 40$ B-dwarfs on isotropic orbits inside 1" (the "S-cluster"). Center: the GMCs and molecular clumps of the circumnuclear disk dramatically shorten the relaxation time outside $r_{h}$ (blue and green lines, for two limiting assumptions about the masses of the GMCs), relative to that by stars alone (red line), and also reduce from afar the relaxation time inside $r_{h}$ (circles) [4,8]. Right: possible signature of RR in the inner pc of the GC [9]. Significant relaxation is expected where the relaxation times are shorter than the typical ages of the different dynamical component. 2-body relaxation is faster than scalar ( $J$-changing) $\mathrm{RR}$ only far from the MBH, where the enclosed stellar mass becomes substantial, or extremely close to the MBH, where GR precession is strong. The two lines for scalar RR represent different assumptions about the mean stellar mass. Scalar RR is an important process for the dynamics of GW sources, and may be also implicated in the randomization of eccentricities of the S-stars [6,7] and the relaxed giants. Vector $(\hat{\boldsymbol{J}}$-changing) RR is everywhere faster than 2-body relaxation, as long as the average potential is close to spherical, Vector RR may explain the inner cutoff of the young star disk, and the randomization of the orbital inclinations of the relaxed giants. (reproduced with permission from the Astrophysical Journal).

field of an $\mathrm{MBH}$, or interactions of stars with a circumnuclear gas disk or star disk around a MBH. All of the above can also be relevant when the $\mathrm{MBH}$ is not stationary in the center of the galaxy, but is recoiling following an asymmetric coalescence event, or in the case where the stars surround a binary MBH (Sec. 4.2). Many of these supply channels are discussed in detail elsewhere in these proceedings.

\section{RAPID RELAXATION MECHANISMS}

TD rates are determined by the supply channel, and by the replenishment rate of the LC. 2-body relaxation is unavoidable, and thus sets a guaranteed minimal replenishment rate. However, other processes can contribute, or even dominate relaxation. Much of the motivation for the study of these processes, and most of the empirical tests of these ideas, are due to the unique observations of stars in the Galactic Center (GC), very close to the Galactic $\mathrm{MBH}$ $\left(\mathrm{SgrA}^{\star}\right)$ [2,3] (Fig. 2L). I briefly describe here rapid relaxation by massive perturbers (MPs), by resonant relaxation (RR), and orbital evolution in non-spherical systems.

\subsection{Massive perturbers}

The rate of relaxation of a star of mass $M_{\star}$ by 2-body encounters with perturbers of number density $n_{p}$ and mass $M_{p}$ can be estimated by a simple " $\Gamma \sim n v \Sigma$ " collision rate calculation. Approximating the relative velocity $v$ by the velocity dispersion $\sigma$, and the interaction cross-section $\Sigma=\pi R^{2}$ with the collision radius $R \sim G M_{p} / \sigma^{2}$ (the closest approach needed for an $O(1 \mathrm{rad})$ angular deflection) yields a relaxation rate of $\Gamma \propto n_{p} M_{p}^{2} / \sigma^{3}$ (integration over collisions at larger separations increases the rate by the Coulomb factor). The $M_{p}^{2}$ dependence of the rate implies that even a small density of very massive objects can dominate 2-body relaxation by stars, when $n_{p} M_{p}^{2} / n_{\star} M_{\star}^{2} \gg$ 1 [4]. This is indeed the case in many galactic nuclei, where giant molecular clouds (GMCs), stellar clusters (and possibly IMBHs) lower the estimated 2-body relaxation time by orders of magnitude relative to that due to stars alone.

The acceleration of the relaxation rate in the GC, estimated from observations of GMCs, is dramatic outside $r_{h}$, where GMCs are found, and substantial also inside $r_{h}$ (Fig. 2C). Since on the $r \sim r_{h}$ scale, where most of the TD flux originates (Sec. 2; Fig. 1L), the LC can already be filled by stellar 2-body relaxation alone, MPs raise the TD rate only by $\times 3$ over that expected by stars alone (the effect is mostly due to the less massive gas clumps at $\sim r_{h}$. This conclusion could overturn if IMBHs do exist inside $r_{h}$ ).

The most dramatic effect of MPs is on the scattering of binaries from outside $r_{h}$ to LC orbits that lead to their tidal separation (not to be confused with star-destroying TD), resulting in the capture of one of the stars on a tight eccentric orbit around the $\mathrm{MBH}$, and the ejection of the other out of the Galaxy as a hyper-velocity star [5]. A comparison of the numbers of bound B-dwarfs observed in the inner 1" of the GC (the "S-stars", see Fig. 2L), and the numbers of hyper-velocity stars (also mostly B-dwarfs), supports this scenario for S-star capture by MP scattering [4]. While the capture process itself is "dark", the captured stars are so close to the $\mathrm{MBH}$, that a substantial fraction of them are likely to eventually undergo luminous TD [6,7] (Sec. 5). 


\subsection{Resonant relaxation}

$\mathrm{RR}$ is a rapid process of angular momentum relaxation $[9,10]$, which takes place in near-spherically symmetric potentials where orbital evolution is limited (e.g. fixed ellipses in a Kepler potential; fixed orbital planes in a general spherical potential). In that case, on timescales much longer than $P$, but still shorter than $t_{\mathrm{coh}}$, the time it takes for perturbations to grow and break the coherence of the symmetry (e.g. deviations from a Kepler potential due to the mass of the stars themselves, or due to GR precession), the averaged orbits of the field stars can be viewed as fixed mass wires (in a Kepler potential), or fixed annuli (time-averaged rosettes in a spherical potential).

The potential of this near-stationary background mass distribution conserves orbital energy, but the finite number of stars means that there are order $\sqrt{N_{\star}}$ fluctuations from isotropy, which give rise to near-constant residual torques that rapidly $(\propto t)$ change the angular momentum of a test star. The accumulated change over a coherence time, $(\Delta J)_{\mathrm{coh}} \sim\left(\sqrt{N_{\star}} G M_{\star} / R\right) t_{\mathrm{coh}}$, where $R$ is the typical size of the test star orbit, then becomes the mean free step size of an accelerated random walk, which can be written as $\left(\Delta J / J_{c}\right)(t)=\sqrt{t / t_{R R}}$ (for $\left.t>t_{\mathrm{coh}}\right)$, where the RR timescale is $t_{R R} \sim Q^{2} P^{2} /\left(N_{\star} t_{\mathrm{coh}}\right)$.

The evolution of the angular momentum then depends on the symmetries of the potential and the process that sets the shortest coherence time. In a near-Kepler potential, all components of the angular momentum are torqued, and in particular RR can change the magnitude $J$, and drive a wide orbit into a near radial one that lies inside the LC. The relevant coherence-limiting (quenching) mechanisms are precession due to the enclosed stellar mass far from the $\mathrm{MBH}$, and GR in-plane precession near it (Fig. 2R). This variant of RR is sometimes called "scalar RR". In addition, any spherical potential supports a restricted form of RR, where the time-averaged mass annuli change only the inclination of the orbital plane of a test star, $\hat{J}=J / J$, but not the magnitude $J$. In the absence of axial symmetry breaking, this is an extremely fast process, since it is only quenched by the orbital randomization due to RR itself $^{2}$. The self-quenching timescale $t_{\text {coh }}^{s q}$ is then set by the requirement $(\Delta J)_{\text {coh }} \sim J_{c}$, which is a long timescale, $t_{\text {coh }}^{s q} \sim Q P / \sqrt{N_{\star}}$. This variant of RR is sometimes called "vector RR".

RR is easily detected in $N$-body simulations [11], and there is also some evidence that it is operating in the GC [9] (Fig. 2R). Its direct effect on the integrated TD rates is not large (up to $\times 2$ enhancement [10]), since most of the TD flux is from $r \sim r_{h}$, where $N_{\star} M_{\star} \sim M_{\bullet}$ and orbital precession due the enclosed stellar mass quenches RR. However, RR may play a more significant role for TD events by stars on tight orbits (Sec. 5).

\subsection{Non-spherical systems}

A high level of symmetry in the stellar potential is associated with conserved quantities, which restrict orbital

\footnotetext{
2 The separation of background stars/test star is arbitrary-all the stars in the system are simultaneously torqued by RR.
}

evolution and can slow down LC refilling to the minimal rate provided by 2-body relaxation (a collisional effect outside the domain of potential theory). Conversely, less symmetry leaves more freedom for orbits to evolve, and opens new possibilities for efficient replenishment of losscone orbits.

Axisymmetric systems have centrophilic (center approaching) orbits, which lead to a $\times 2$ enhancement in the TD rate over that predicted for spherical systems [12]. Another consequence is the higher incidence of deep TDs, with very small periapse distance, which may result in tidal detonation (Sec. 4.3). Triaxial systems can support a large fraction pf chaotic orbits, which can lead to a dramatic $\times 10-100$ enhancement while the chaotic orbits persist [13]. This conclusion depends however on whether chaotic orbits can exist in the presence of the stabilizing influence of the central MBH.

\section{TIDAL DISRUPTION RATES}

\subsection{Single MBH in steady state}

The basic configuration to consider is TD on a single MBH surrounded by stellar cluster in steady state. TD rate estimates for such a configuration have a long history, starting in the mid 1970's. Early analyses focused on the similar process of a star disrupted by an IMBH in a globular cluster $[14,15]$. The first estimate for TDs on a $\mathrm{MBH}$ in a galactic nucleus yielded a very high rate, of $10^{-3}-10 M_{\odot} \mathrm{yr}^{-1} \mathrm{gal}^{-1}$ [16]. Contemporary rate estimates, with more careful modeling of the process, yield consistently lower values. These are summarized in table 1.

\subsection{Binary MBHs beyond steady state}

The near ubiquity of nuclear MBHs from very early cosmic times, and the bottom-up structure formation paradigm, together imply that $\mathrm{MBH}$ pairs and bound $\mathrm{MBH}$ binaries must be a commonly occurring, probably transient phase in galaxy evolution. Recent studies have found that hard, high mass ratio binary MBHs are efficient disruptors. This can come about by Kozai forcing of stellar orbits around the primary (more massive) $\mathrm{MBH}$, induced by the secondary MBH [24], or by chaotic mixing due to the secondary [25]. These effects can lead to a transient phase of very high TD rates, $\lesssim 1 \mathrm{yr}^{-1}$, and account overall for $O(10 \%)$ of the steady state cosmic TD rate. Another consequence of this high TD rate is the possibility of multiple events from the same galaxy over an observationally relevant short timescale, which could provide a signature of a binary MBH [26].

Certain combinations of orbital angular momentum and $\mathrm{MBH}$ spin orientations lead to a strong asymmetry in the emitted GW flux and a very fast recoil velocity for the newly coalesced $\mathrm{MBH}$ [27]. The recoiling $\mathrm{MBH}$ retains a small cluster of tightly bound stars with it. The recoil can promptly refill the LC and briefly raise the TD rate to $O\left(0.1 \mathrm{yr}^{-1}\right)$, thereby providing a prompt EM counterpart to 
Table 1. Contemporary TD rate estimates for a single $\mathrm{MBH}$ in steady state.

\begin{tabular}{llll}
\hline Special physical features & ${\mathrm{Rate}\left[\mathrm{yr}^{-1} \mathrm{gal}^{-1}\right]}^{-1}$ & Comments & References \\
\hline- & $10^{-6}-10^{-4}$ & & {$[1]$} \\
Axiality & $10^{-9}-10^{-4}$ & & {$[12]$} \\
Triaxiality/Chaos & $\lesssim 10^{-2}$ & & {$[13]$} \\
Resonant relaxation & - & $\times 2$ enhancement only & {$[10,17]$} \\
Revised $M_{\bullet} / \sigma$ relation & - & $\times 10$ increase over previous estimates & {$[18]$} \\
Infall/inspiral & $9 \times 10^{-5}$ & Rate estimate for the GC & {$[19]$} \\
$N$-body experiments & $10^{-6}-10^{-4}$ & & {$[20]$} \\
MBH spin & - & Higher TD rate by increased max $M \bullet$ & {$[21-23]$} \\
\hline
\end{tabular}

the GW signal of the coalescence [28]. Much later, as the recoiling $\mathrm{MBH}$ moves farther away from the center of the galaxy, the small dense cluster it carries with it can provide off-center or even extra-galactic TD events at rates between $0.01[29]$ to $\lesssim 1[30]$ of those of non-recoiling MBHs.

\subsection{TD-related exotica}

It is interesting to note that there are exotic processes that must occur, and at substantial rates, in association with TD. These happen when the orbital periapse is either just outside the tidal disruption radius $r_{t}$, or very deep inside it.

Near misses. Stars that are deflected from a tight enough orbit to an eccentric one with periapse within $\sim 2 r_{t}$ can enter an inspiral phase, similar to that of GW inspiral events, as "squeezars" [31], stars that brightly shine by the orbital energy dissipated as they tidally interact with the MBH. This inspiral-type mechanism has a rate that is typically $\sim 0.05$ of the TD rate, $0.1-1 \mathrm{yr}^{-1}$ in the GC (Sec. 2.2). When stars are not captured on an inspiral orbit, they undergo a single very strong tidal scattering interaction [32], which can spin them up and mix them, thereby affecting their subsequent spectroscopic signature and evolution. Such event are predicted to occur at $O(1)$ of TD rate, $10^{4}-10^{5} \mathrm{yr}^{-1}$ in GC. Finally, the extended, low-gravity envelopes of giant stars are susceptible to tidal stripping, leaving behind the hot bare cores [33]. These events are estimated to occur at $\sim 0.01$ of the TD rate in the GC.

Direct hits. When the periapse of the incoming star is $\ll r_{t}$, and the stellar core is in a state that is favorable to fast nuclear burning processes (post-hydrogen burning), the strong tidal pancake compression the star experiences as it crosses periapse can lead to a supernova-like tidal detonation event $[34,35]$. The rate of such events is estimated at $<0.1$ of the TD rate.

\section{TIDAL DISRUPTIONS FROM TIGHT ORBITS}

Standard incoherent 2-body relaxation results in losscone refilling that is dominated by stars from typical radii $r \sim r_{h}$, where the dynamical timescale is long, the $\mathrm{MBH}$ potential no longer dominates (and therefore RR is inefficient), and GR effects are completely negligible. However, there are compelling reasons to believe that there are situations where stars on much tighter orbits can contribute significantly to the total TD rate. This can be the case, for example, when there is substantial in-situ star formation in gravitationally unstable accretion disks, as is observed in the GC on the $O\left(0.1 r_{h}\right)$ scale [36]; or for stars captured around the $\mathrm{MBH}$ by the tidal separation of binaries [37] (Sec. 3.1); or for stars that are tightly bound to a recoiling $\mathrm{MBH}[29,30]$.

In such cases, dynamical mechanisms that have not been considered before may come into play. I focus here on two newly uncovered effects: stellar relaxation by anomalous diffusion [38], and the Schwarzschild Barrier (SB) in phase space [39]. While it is too early to tell whether these effects have a significant impact on TD events, their recent discovery highlights the possibility that basic key components may still be missing from our understanding of dynamics near MBHs.

Anomalous diffusion. Normal diffusion can be viewed as a random walk process, where the accumulated change $\Delta E$ or $\Delta J$ grows with time as $\propto \sqrt{t}$ (I omit here for brevity discussion of drift). This is a consequence the Central Limit Theorem (CLT) applied to multiple scattering events. The CLT is valid since each of those is physically limited to some finite range of $\Delta E$ or $\Delta J$, and is thus well-defined statistically. However, the rate of convergence to the CLT can be very slow, depending on how nearly-divergent is the physics of the scattering. In that case, on timescales shorter than CLT convergence, the evolution of $E$ or $J$ is by anomalous diffusion. The rate can be very different from $\sqrt{t}$; it may depend on $\Delta E$ itself (non-self-similar evolution); it can result in improbably high rates of "high- $\sigma$ " events (when mis-interpreted as Normal probability events). The Newtonian gravitational force with its formal divergence at $r_{12} \rightarrow 0$ is exactly such a special case (Fig. 3L). On timescales shorter than $\sim 0.1 t_{E}$ there are substantial deviations from the expected statistics of normal diffusion.

Anomalous diffusion may be particularly relevant close to the $\mathrm{MBH}$, where it could change the branching ratios between infall and inspiral (effectively changing $r_{\text {crit }}$, see Sec. 2.2). This can be seen by noting that inspiral can occur only when the dissipation timescale is shorter than the relaxation time, $t_{\text {diss }} \sim\left(E / \Delta E_{\text {diss }}\right) P<t_{J}\left(J_{l c}\right)$, where $\Delta E_{\text {diss }}$ is the energy dissipated per peripassage, and where both $\Delta E_{\mathrm{diss}}$ and $t_{J}$ are evaluated close to the LC boundary. 

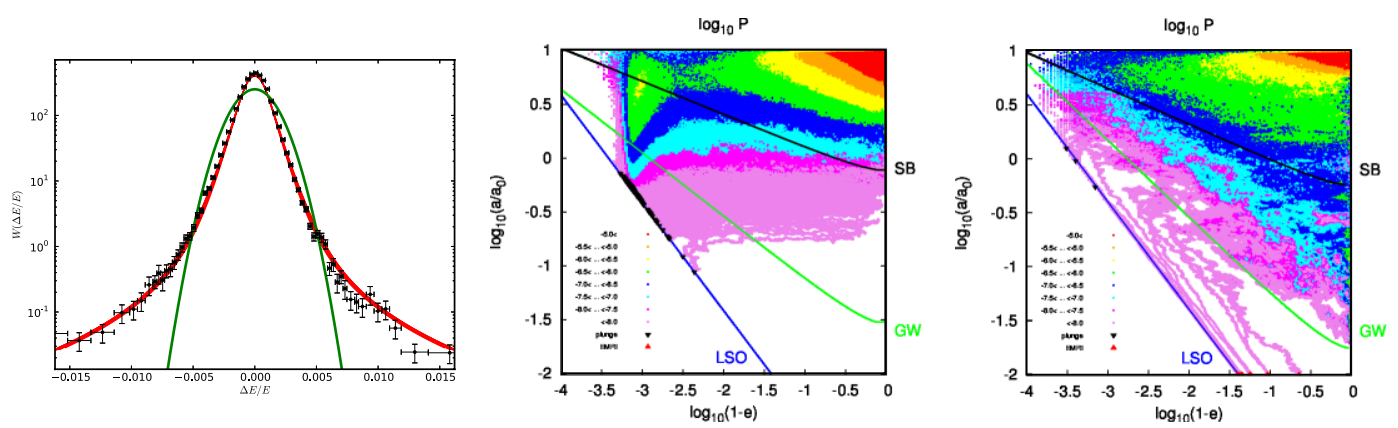

Figure 3. Previously unconsidered dynamical effects that could modify TD rates from orbits near the MBH. Left: anomalous diffusion on timescales shorter than $t_{E}$ leads to faster than $\propto \sqrt{t}$ evolution in phase space (crosses from $N$-body simulation and theory in red trace the relative change in energy $\Delta E / E$ ), and in particular makes "high- $\sigma$ " events much more common than they would be in normal diffusion (Gaussian probability distribution function in green) [38]. Center: the phase-density of $50 M_{\odot}$ stellar mass "black holes" around a $M_{\bullet}=10^{6} M_{\odot} \mathrm{MBH}$ in $(\log [1-e], \log a / 1 \mathrm{mpc}) \sim(\log J, \log E)$ phase space, as obtained by Monte Carlo experiments [40]. In the absence of GR, RR rapidly throws all stars into the MBH across the last stable orbit (LSO). Right: with GR in-plane precession, the Schwarzschild Barrier (a resonance between the RR torques and the GR precession) [39] deflects stars back to lower eccentricity orbits and allows gradual GW inspiral to take place (see tracks with nearly constant $r_{p}$ parallel to the LSO line).

Since $t_{\text {diss }} / t_{J}\left(J_{l c}\right) \propto N_{\star} \propto a^{3-\alpha}$ for an $n_{\star} \propto r^{-\alpha}$ cusp (Sec. 2.1), then if anomalous diffusion in $\Delta J$ (not yet explored) leads to overall faster $J$-relaxation, it will shift $r_{\text {crit }}$ to a smaller radius, implying more prompt TDs and fewer tidal inspirals than estimated for normal diffusion.

The Schwarzschild Barrier. RR torquing of orbits (Sec. 3.2; Fig 2R) becomes more important closer to the $\mathrm{MBH}$, until it is quenched by GR in-plane precession very close to it. It was hypothesized that this quenching is crucial for "saving" stars from plunging into the $\mathrm{MBH}$, and allowing them to inspiral gradually (Fig. 3C). Relativistic $N$-body simulations [39] revealed that the GR precession not only quenches RR and enables inspiral, but in fact creates a reflective barrier in phase space, which can be approximately understood as arising from a resonance between the GR precession frequency and the rate of angular momentum change due to the RR torques (Fig. 3R). Stars can cross the SB only by 2-body scattering. Once past the barrier, they are free to inspiral. Current analysis of the SB phenomenon has focused only of GW inspiral. The implications for TD events from tight orbits are still unknown.

\section{SUMMARY}

It is clear that there are multiple plausible channels by which stars can be deflected into LC orbits that lead to TD events. However, quantitative estimate of the cosmic rates of such events are severely limited by astrophysical uncertainties. On the theoretical side, there is a clear need for a better understanding of the conventional dynamical mechanisms that are known to play part in the replenishment of the $\mathrm{LC}$, and there are also new, recently discovered mechanisms that need to be taken into account. In spite of these uncertainties, it seems quite realistic to expect that TD rates should be high enough to be observationally interesting. Indeed there are multiple efforts to approach the problem empirically, and detect TD events (see in these proceedings).
As is evident from this review, the dynamics of TD events are tightly linked to those of GW sources through the infall/inspiral connection. The study of one bears directly on the other. One additional important connection is due to the unfortunate fact that the electromagnetic signature of TD events (discussed elsewhere in these proceedings) may not be distinct enough from other types of AGN variability to offer definitive identification. This may ultimately be achieved only by the detection of lowfrequency GW counterparts to TDs.

This work was supported by ERC Starting Grant No. 202996 and DIP-BMBF Grant No. 71-0460-0101.

\section{References}

[1] D. Syer, A. Ulmer, MNRAS 306, 35 (1999)

[2] T. Alexander, Phys. Rep. 419, 65 (2005)

[3] R. Genzel, F. Eisenhauer, S. Gillessen, Reviews of Modern Physics 82, 3121 (2010)

[4] H.B. Perets, C. Hopman, T. Alexander, ApJ 656, 709 (2007)

[5] W.R. Brown, M.J. Geller, S.J. Kenyon, M.J. Kurtz, ApJ 622, L33 (2005)

[6] H.B. Perets, A. Gualandris, G. Kupi, D. Merritt, T. Alexander, ApJ 702, 884 (2009)

[7] B. Bar-Or, T. Alexander (2012), in preparation

[8] T. Alexander, in The Galactic Center: a Window to the Nuclear Environment of Disk Galaxies, edited by M.R. Morris, Q.D. Wang, F. Yuan (2011), Vol. 439 of ASP Conf. Ser, p. 129

[9] C. Hopman, T. Alexander, ApJ 645, 1152 (2006)

[10] K.P. Rauch, S. Tremaine, New Astronomy 1, 149 (1996)

[11] E. Eilon, G. Kupi, T. Alexander, ApJ 698, 641 (2009), 0807. 1430

[12] J. Magorrian, S. Tremaine, MNRAS 309, 447 (1999)

[13] D. Merritt, M.Y. Poon, ApJ 606, 788 (2004)

[14] J. Frank, M.J. Rees, MNRAS 176, 633 (1976)

[15] H. Cohn, R.M. Kulsrud, ApJ 226, 1087 (1978) 
[16] P.J. Young, G.A. Shields, J.C. Wheeler, ApJ 212, 367 (1977)

[17] K.P. Rauch, B. Ingalls, MNRAS 299, 1231 (1998)

[18] J. Wang, D. Merritt, ApJ 600, 149 (2004)

[19] T. Alexander, C. Hopman, ApJ 590, L29 (2003)

[20] M. Brockamp, H. Baumgardt, P. Kroupa, MNRAS 418, 1308 (2011)

[21] S. Kobayashi, P. Laguna, E.S. Phinney, P. Mészáros, ApJ 615, 855 (2004)

[22] P.B. Ivanov, M.A. Chernyakova, A\&A 448, 843 (2006)

[23] M. Kesden, Phys. Rev. D 85, 024037 (2012)

[24] P.B. Ivanov, A.G. Polnarev, P. Saha, MNRAS 358, 1361 (2005)

[25] X. Chen, A. Sesana, P. Madau, F.K. Liu, ApJ 729, 13 (2011)

[26] C. Wegg, J. Nate Bode, ApJ 738, L8 (2011)
[27] J.D. Schnittman, A. Buonanno, ApJ 662, L63 (2007)

[28] N. Stone, A. Loeb, MNRAS 412, 75 (2011)

[29] N. Stone, A. Loeb, MNRAS 422, 1933 (2012)

[30] S. Komossa, D. Merritt, ApJ 683, L21 (2008)

[31] T. Alexander, M. Morris, ApJ 590, L25 (2003)

[32] T. Alexander, M. Livio, ApJ 560, L143 (2001)

[33] M.B. Davies, A. King, ApJ 624, L25 (2005)

[34] B. Carter, J.P. Luminet, Nature 296, 211 (1982)

[35] P. Laguna, W.A. Miller, W.H. Zurek, M.B. Davies, ApJ 410, L83 (1993)

[36] T. Paumard et al., ApJ 643, 1011 (2006)

[37] J.G. Hills, Nature 331, 687 (1988)

[38] B. Bar-Or, G. Kupi, T. Alexander, Submitted to ApJ. ArXiv e-prints (2012), 1209.4594

[39] D. Merritt, T. Alexander, S. Mikkola, C.M. Will, Phys. Rev. D 84, 044024 (2011)

[40] T. Alexander, D. Merritt (2012), in preparation 